\title{
Die Zauberflöte : tradition et reflets
}

Die Zauberflöte: Tradition und Abbilder

Die Zauberflöte: Tradition and reflections

\section{Manuel Durand-Barthez}

\section{(2) OpenEdition}

\section{Journals}

Édition électronique

URL : http://journals.openedition.org/ceg/431

DOI : $10.4000 /$ ceg. 431

ISSN : 2605-8359

Éditeur

Presses Universitaires de Provence

Édition imprimée

Date de publication : 5 avril 2017

Pagination : 171-184

ISBN : 979-10-320-0103-5

ISSN : 0751-4239

Référence électronique

Manuel Durand-Barthez, "Die Zauberflöte : tradition et reflets », Cahiers d'Études Germaniques [En ligne], 72 | 2017, mis en ligne le 05 octobre 2018, consulté le 27 novembre 2020. URL : http:// journals.openedition.org/ceg/431 ; DOI : https://doi.org/10.4000/ceg.431 


\title{
Die Zauberflöte
}

\section{Tradition et reflets}

\section{Manuel DURAND-BARTHEZ}

\author{
Université Toulouse Jean-Jaurès, École nationale des chartes
}

Trahison, transmission, la tradition se ressource continûment. Elle marque la dramaturgie de la Zauberflöte en puisant ses origines dans plusieurs catégories d'actions réflexives ${ }^{1}$. D'abord celle du modèle qui invoque un personnage $d u$ théâtre populaire, héritier des facéties médiévales: Hans Wurst, dont Papageno est un avatar. Ensuite la catégorie de l'imitation lorsque des rituels inspirés par la mythologie copient, avec la déformation du reflet et de l'interprétation, l'esprit et la gestuelle décrits chez les auteurs antiques. Nous nous attacherons à examiner comment ces rituels ont jalonné le chemin des rebelles ou des initiés; ivresse ou sagesse répondent aux mêmes incantations et aux mêmes canons. Les individus semblent soumis à une prédestination fatale et canonique, les uns demeurent spirituellement écartelés et diminués à l'instar de l'oiseleur, prédateur captif de sa propre fonction, les autres ont vocation à s'élever, se pliant à un modèle conçu au royaume de l'Un. Cette dichotomie affecte aussi les genres puisque les profanes postulantes sont marginalisées dans le rituel maçonnique dit d'Adoption, issu d'une interprétation spécieuse de la religion égyptienne, tributaire des chroniques tardives d'historiens antiques, profondément teintés de misogynie.

\section{Hans Wurst et Mozart}

Lors d'une conférence qu'il prononça le 16 mars 2005 à la Maison des sciences de l'homme de Paris sur La figure du Hanswurst dans la littérature et la culture autrichiennes, Gerald Stieg affirmait: "Papageno est l'une des multiples

1. Sur le livret de La Flûte enchantée, on pourra se reporter aux études détaillées de Jan Assmann, (Die Zauberflöte. Oper und Mysterium, Carl Hanser Verlag, München / Wien 2005), dans lesquelles on trouve une étude serrée du personnage de Papageno, une analyse des mystères égyptiens dans leur réception par une franc-maçonnerie à ambition scientifique ainsi qu'une présentation de la conception des mystères dans les loges maçonniques à l'époque de Mozart, et Dieter Borchmeyer (Mozart, oder die Entdeckung der Liebe, Frankfurt am M., Insel, 2005) ainsi qu'à deux numéros savants de L'Avant-Scène Opéra (La Flûte enchantée, $\mathrm{n}^{\circ} 1,1^{\mathrm{er}}$ janvier 1976 et $n^{0} 196,15$ octobre 2009). 
métamorphoses du personnage de Hans Wurst ${ }^{2}$." De fait, les épisodes lors desquels s'illustre l'oiseleur évoquent à certains égards l'Autrichien Josef Anton Stranitzky (1676-1726) sous les traits du Hanswurst (ce comédien était devenu emblématique du rôle au début du XVIII ${ }^{\mathrm{e}}$ siècle). Hans Wurst est un avatar du diable tel qu'il apparaissait dans les facéties et mystères du Moyen Âge. Il " casse " la sagesse, suscite en elle une dichotomie fatale, c'est la personnification du deux (et du diabolein) qui traverse, coupe et perturbe. Il est imperméable à l'éducation, aux bonnes manières, il remet toujours tout en question. Incapable de sérieux, il est naturellement comique. Sur scène, Hans Wurst sera souvent le serviteur des grands: Achille, Cicéron, César. Autour d'eux, il sème le trouble en faisant rire le peuple. Et cela ne plaît guère à la haute société qui honnit ce théâtre. L'Allemagne, plus celle de Gottsched que de Lessing, le disqualifiera, l'exclura de son espace culturel, à la différence de ces méridionaux moins rigoureux que sont les Autrichiens, à l'exception notable de Joseph von Sonnenfels :

Les principaux griefs faits à Hanswurst et Bernardon depuis Gottsched jusqu'à Sonnenfels [...], tiennent en quelques mots: vulgarité, obscénité, mauvais goût, violence, Hanswurst apparaissant comme un monstre d'immoralité doublé d'un repoussoir pous un public viennois appelé à être éduqué, pour reprendre la formule de Gottsched, " selon les règles de la raison ${ }^{3} "$.

À l'époque de la Flûte enchantée, la franc-maçonnerie représente un corps social majoritairement élitiste, qui se veut représentatif d'une société évoluée. Si la sagacité du spectateur remet le personnage de Papageno "à sa place ", mal nécessaire en quelque sorte pour mieux valoriser Tamino, alors la Flûte enchantée peut s'insérer dans les grands programmes d'éducation du genre humain en honneur au XVIII $I^{\text {e }}$ siècle. Néanmoins, souligne G. Stieg, "les sympathies de Mozart sont très nettement aussi du côté de Papageno ${ }^{4}$ ". Celui-ci calque le caractère ouvertement débridé du compositeur, apte à relativiser, à se distancier vis-à-vis de la rigidité excessive d'une société dominante. La Flûte enchantée reflète la personnalité de Mozart, à la fois Papageno et Tamino. Par conséquent - et c'est l'un des traits marquants de cette œuvre - le spectateur est souvent désemparé face à cette ambiguïté. Dans une perspective à peu près similaire, Goethe avait aussi laissé la part belle (mais passablement cachée) au modèle de Papageno, dans Hans Wurst Hochzeit oder der Lauf der Welt - Ein mikrokosmisches Drama (1775). À ce propos, les commentateurs de la Flûte enchantée ont fréquemment cité Goethe dans les propos qu'il tint à Johann Peter Eckermann au sujet d'Helena:

2. Gerald Stieg, La figure du Hanswurst dans la littérature et la culture autrichiennes, première conférence Portrait du Hanswurst, Entretien avec Peter Stockinger, Paris, Éditions de la Maison des sciences de l'homme, en ligne: [http://www.archivesaudiovisuelles.fr/419/], consulté le 23 novembre 2015.

3. Marc Lacheny, Littérature " d'en haut ", littérature "d'en bas "? La dramaturgie allemande et le théâtre populaire viennois de Stranitzky à Nestroy, Berlin, Frank \&t Thimme, 2016, p. 42.

4. Ibid. 
Aber doch, sagte Goethe, ist alles sinnlich und wird, auf dem Theater gedacht, jedem gut in die Augen fallen. Und mehr habe ich nicht gewollt. Wenn es nur so ist, daß die Menge der Zuschauer Freude an der Erscheinung hat; dem Eingeweihten wird zugleich der höhere Sinn nicht entgehen, wie es ja auch bei der Zauberflöte und andern Dingen der Fall ist. ${ }^{5}$

Cette ambivalence est ancienne; elle empreint les religions de l'Antiquité en leur conférant une double destination. Le polythéisme est le jouet du peuple, noyé dans l'ivresse du multiple. Tant qu'il s'y complaît, l'élite peut approfondir au calme les mystères de l'Un. Mais la rigueur morale qu'implique celui-ci peut inciter ses adeptes à rejoindre les humbles, non par facilité, mais précisément par humilité, pour mettre périodiquement à distance la sphère des privilégiés, au nom enfın d'une certaine " hygiène de vie " qui aère l'esprit dans un hédonisme plus ou moins voulu. C'est exactement le cas de Mozart, dont on connaît les frasques, l'exubérance, les dérapages scatologiques et les sorties obscènes. Sur un plan strictement formel, il sera par exemple souvent laxiste avec l'orthographe dont l'imperfection, peut-être entretenue, l'amènera complaisamment à irriter son père. C'est pourquoi Gerald Stieg évoque la sympathie du compositeur à l'égard de Papageno dont le rôle et le parcours (ou la stagnation ?) occupent une place non négligeable dans le Singspiel. L'homme-oiseau, dont nous analyserons plus loin les caractéristiques, fait partie intégrante du monde. Si l'ambivalence prend ses racines dans un modèle antique et religieux, il faudra examiner comment l'Égypte a inspiré, à travers les récits d'historiens antiques tardifs (Hérodote, Plutarque, Diodore de Sicile...), les éminences intellectuelles de la Maçonnerie européenne de la fin du XVIIII siècle.

\section{L'Urbild égyptien}

Les symboles maçonniques qui fleurissent le frontispice de l'édition originale du livret de la Zauberflöte, œuvre du graveur (autre Frère) Ignaz Alberti ${ }^{6}$, apparaissent dans un décor assez hétéroclite dont l'égyptianité est soulignée par la présence d'un obélisque (ou d'une pyramide, dont les proportions auraient été singulièrement rétrécies) frappé de hiéroglyphes et de figures issues de la mythologie égyptienne (comme l'ibis et le taureau Phtah). Au fond, la façade plutôt dépouillée d'un édifice monumental, ceint d'arcades, ne présente aucun des réels signes distinctifs susceptibles de lui attribuer une destination religieuse. À droite, une urne imposante chargée de symboles sculptés, au pied de laquelle sont rangés divers outils et accessoires associés à l'univers maçonnique. Un corps humain, semble-t-il inanimé gît à terre, dont le visage est tourné vers l'obélisque

5. Johann Peter Eckermann, Gespräche mit Goethe in den letzten Jahren seines Lebens, Leipzig, Brockhaus, 1836, vol. I, p. 317.

6. En ligne: [https://commons.wikimedia.org/wiki/File:Die_Zauberflöte,_Ignaz_Alberti.jpg] (consulté le 23 novembre 2015), in Emmanuel Schikaneder, Die Zauberflöte: eine grosse Oper in zwey Aufzügen; die Musik ist von Herrn Wolfgang Amade Mozart, Kapellmeister, und wirklichem $k . k$. Kammer-Compositeur, Wien, Ignaz Alberti, 1791. Les extraits cités proviennent de cette édition originale. 
ou l'acacia sacré qui le surplombe ${ }^{7}$. Une étoile à cinq branches est suspendue à la voûte dont la courbure n'est pas franchement visible, tant la pénombre est pesante. Parmi les multiples indications de décor dans le livret, celle-ci semble se rapprocher le plus de cette description sommaire du frontispice:

Das Theater verwandelt sich in einen kurzen Vorhof des Tempels, wo man Rudera ${ }^{8}$ von eingefallenen Säulen und Pyramiden sieht, nebst einigen Dornbüschen. An beyden Seiten stehen practicable hohe altägyptische Thuren, welche mehr Seitengebäude vorstellen (Acte II, scène 2).

Cette illustration fit l'objet de multiples gloses qu'il ne nous appartient pas de développer ici. Le contexte maçonnique de ces images mérite un rappel sommaire de son impact sociétal en Autriche à l'époque de Mozart. François I ${ }^{\text {er }}$, époux de Marie-Thérèse, alors duc de Lorraine, fut reçu Frère dans une loge de La Haye en 1731 et formé par un ambassadeur d'Angleterre auprès du Prince d'Orange. Malgré les réticences de l'archiduchesse, la Maçonnerie put prospérer jusqu'à l'avènement de son fils Joseph II. Celui-ci promulgue une ordonnance le 24 décembre 1785 qui réduisait de façon drastique le nombre de loges, étroitement surveillées. De huit, le nombre d'Ateliers viennois passe à trois puis à deux; d'un millier, les Frères viennois sont réduits à 360. Ce nonobstant, l'empereur n'est pas foncièrement opposé à la Maçonnerie, il souhaite simplement éviter ses débordements 9 .

C'est donc dans un contexte plutôt mouvementé sur le plan de l'histoire maçonnique que Mozart compose la Flûte enchantée. Il serait excessif d'évoquer une période marquée par la censure dont les effets sur les Maçons se firent plutôt sentir au début du XIX ${ }^{\mathrm{e}}$ siècle. Néanmoins, on devine dans les propos de Goethe comme dans la trame de la Zauberflöte une propension, sinon à dissimuler, $\mathrm{du}$ moins à voiler un message sur lequel le lecteur-spectateur est conduit à s'interroger. Pour reprendre les propos d'Eckermann à propos de l'Helena de Goethe dans la partie moderne et romantique:

denn eine halbe Weltgeschichte steckt dahinter; die Behandlung ist bei so großem Stoff nur andeutend und macht sehr große Ansprüche an den Leser ${ }^{10}$.

À l'élite, une tendance au monothéisme, une recherche de l’Un, un dépassement de la dyade évoquée en introduction à propos de Hans Wurst et de la représentation comique du diable. Au diabolein se substitue le sunbolein. C'est ce dernier qui

7. Arbre osiriaque et solaire; de son essence serait issu Horus.

8. Rudera : pluriel du neutre Rudus : décombres, ruines. Fait songer au décor de quelques jardins romantiques à l'image du parc Monceau à Paris, collection lapidaire en partie issue de ruines monumentales récupérées dans la capitale et " arrangées ", à l'imitation des sites visités par les poètes et artistes de la Renaissance à Rome, par exemple. Ruines évidemment factices par nature dans le contexte du décor de la Flûte.

9. Sur ces événements de l'histoire maçonnique en Autriche, voir: Gustav Kuéss, Bernhard Scheichelbauer, 200 Jahre Freimaurerei in Österreich, Wien, O.Kerry, 1959, p. 65-76; Ludwig Lewis, Geschichte der Freimaurerei in Österreich und Ungarn, Leipzig, C.W. Vollrath, 1872, p. 38-44.

10. Eckermann, Gespräche mit Goethe, vol. 1, p. 317. 
inspire la franc-maçonnerie. La Renaissance avait puisé ses racines chez les Grecs puis les Latins. La Maçonnerie les cherchera plus loin encore, à la source que l'historiographie peut mettre en évidence, certes dans la pénombre, mais avec suffisamment de traces pour pouvoir construire une légende fondatrice: l'Égypte. Qu'elle habite le frontispice du livret apparaît singulièrement significatif. L'incipit imagé du Singspiel est placé par Ignaz Alberti sous le signe des divinités du Nil, à l'ombre de l'Acacia visible en haut de la gravure.

Dans le fil du texte, le décor est clairement situé: "Zwei Sclaven tragen, so bald das Theater in ein prächtiges ägyptisches Zimmer verwandelt ist, schöne Pölster nebst einem prächtigen türkischen Tisch heraus, breiten Teppiche auf " (Acte I, scène 9). On trouve plus loin:

Das Theater ist ein Palmwald; alle Bäume sind silberartig, die Blätter von Gold. 18 Sitze von Blättern; auf einem jeden Sitze steht eine Pyramide und ein großes schwarzes Horn mit Gold gefaßt. In der Mitte ist die größte Pyramide, auch die größten Bäume » (Acte II, scène 1)

Pour clore cette série d'exemples, on peut encore citer le passage suivant: "Das Theater verwandelt sich in das Gewölbe von Pyramiden. Sprecher, und einige Priester. Zwey Priester tragen eine beleuchtete Pyramide auf Schultern " (Acte II, scène 20). Il s'agit en quelque sorte de fonder la Maçonnerie sur des principes antérieurs à la Bible, pour se distinguer du christianisme régnant. Ce phénomène est bien mis en valeur par l'égyptologue Eugène Warmenbol dans sa réflexion sur l'égyptomanie de l'époque:

Plusieurs personnages importants de l'Expédition d'Égypte, tant militaires que civils, étaient francs-maçons: l'intérêt que les loges maçonniques portaient à l'Égypte depuis un siècle en serait un facteur constitutif. Depuis le Sethos de l'abbé Terrasson (1731) ${ }^{11}$, qui trouve un écho dans le Crata Repoa de Karl-Friedrich von Köppen ${ }^{12}$, créateur du rite des Architectes africains (à Berlin en 1767), mais aussi dans les écrits de Giuseppe Balsamo, dit Cagliostro, fondateur du rite de la haute Maçonnerie égyptienne (à Lyon en 1784), l’Égypte passe pour la terre d'origine des francs-maçons ${ }^{13}$.

Dans cette période de l'Aufklärung, on observe ainsi la conception d'une histoire " modelée ", adaptée en quelque sorte dans un acte de copie façonnée pour servir de modèle à l'action maçonnique, mise au service de l'éducation de l'humanité en vue de l'avènement d'un monde libre. Humanité libérée des chaînes de l'Église (associée au pouvoir politique en place) et de la superstition vaincue par la Raison.

On peut aussi rejoindre un autre égyptologue, Jan Assmann, sur le concept de religio duplex, étudié en regard de la civilisation égyptienne dont s'imprégnèrent également les Grecs :

11. Jean Terrasson, Sethos, histoire, ou vie tirée des monumens anecdotes de l'ancienne Égypte, traduite d'un manuscrit grec, Paris, Jacques Guérin, 1731.

12. Karl-Friedrich von Köppen, Crata repoa, oder, Einweihungen in der alten geheimen Gesellschaft der Egyptischen Priester, [S.l. : s.n.], 1785.

13. Eugène Warmenbol, "L'égyptomanie et l'égyptologie en Belgique. Quand passion deviendrait raison ", Bulletin de la Classe des Lettres et des Sciences morales et politiques, Bruxelles, Académie royale de Belgique, 6érie, t. 24, 2013, p. 153-182. 
Die Idee der ägyptischen Religion als religio duplex ist daher weniger das Produkt einer einseitig griechischen Projektion eigener Vorstellungen und Institutionen auf die ägyptische Welt als vielmehr einer gräko-ägyptischen Konfabulation. ${ }^{14}$

Un philosophe platonicien de Cambridge, Ralph Cudworth (1617-1688), propose une définition de la religio duplex. Dans son True intellectual system of the universe (1678), il distingue clairement deux strates sociales en les rattachant à des écoles de pensée.

The Egyptians, besides their vulgar and fabulous theology (which is for the most part that

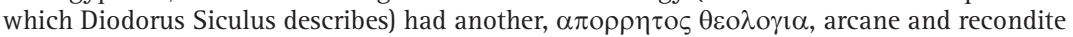
theology - that was concealed from the vulgar, and communicated only to the Kings and such priests and others, as were thought capable thereof. ${ }^{15}$

Ces deux théologies, qui leur étaient propres, présentaient des différences assimilables à celles qui caractérisaient les catégories exotérique et acroamatique ${ }^{16}$ chez Aristote.

$\mathrm{Au}$ XVIII ${ }^{\mathrm{e}}$ siècle, les intellectuels proches de la Maçonnerie estimaient que les Pyramides n'étaient pas seulement des tombes mais plus encore des habitacles confinés dans lesquels les Sages pouvaient se réunir à l'abri de la plèbe afin d'élaborer secrètement les progrès de l'humanité. Le secret protège et favorise la croissance de la plante, à l'abri des adventices.

L'un dépasse le multiple: alors, à la plèbe le polythéisme, les transes et les fêtes vénales.

Le peuple n'aura accès qu'à une version édulcorée, incomplète mais (et cela importe) séduisante des Mystères. La copie, moins précieuse, plus abordable par les sens.

Par ailleurs, l'élite va se rattacher à une mythologie puisée chez Plutarque ${ }^{17}$ et Diodore de Sicile (cité plus haut par Cudworth), historiens tardifs de l'antiquité, mis en avant par la Renaissance. L'élite des Lumières recherche la Lumière en se fondant sur un terrain associant à la fois la vision judaïque du monde (que représente le Temple de Salomon) et la symbolique égyptienne.

14. Jan Assmann, Religio duplex: ägyptische Mysterien und europäische Aufklärung, Berlin, Vlg. der Weltreligionen, 2010, p. 27.

15. "Il est certain qu'à côté de leur théologie destinée au vulgaire et nourrie de légendes (qui pour une très grande part correspondent à celles que décrit Diodore de Sicile), il existait chez les Égyptiens une autre théologie, $\alpha \pi \circ \rho \rho \eta \tau \varsigma$ (secrète) $\theta \varepsilon \circ \lambda \circ \gamma 1 \alpha$, fondée sur des arcanes et maintenue secrète - elle n'était pas révélée au vulgaire, seulement transmise aux rois ainsi qu'aux prêtres et autres personnalités jugées dignes d'en être récipiendaires ", in Ralph Cudworth, The Works of Ralph Cudworth, D.D. containing the True intellectual system of the universe, Sermons Etc., éd. Thomas Birch, Oxford, Talboys, 1829, vol. 2, p. 114.

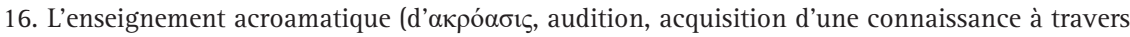
le discours exclusivement oral du maître, sans trace écrite) possède un caractère ésotérique et partant, réservé aux seuls initiés.

17. Johannes Salomon Semler, Joh. Salom. Semlers Erleuterung der egyptischen Altertümer durch Uebersetzung der Schrift Plutarchs von der ,Isis und Osiris“ und der Nachricht von Egypten aus Herodots zweiten Buch mit beigefügten Anmerkungen, Breslau / Leipzig, Johann Jakob Korn, 1748. 
Le peuple est analphabète, l'élite pense et lit. Elle s'appuie sur l'écriture symbolisée par des hiéroglyphes que les contemporains de Mozart ne pouvaient saisir puisque les expéditions napoléoniennes, et plus encore naturellement leur déchiffrage effectué par Champollion (1822) n'eurent lieu qu'après. Ces hiéroglyphes, visibles sur l'obélisque du frontispice d'Alberti, sont là aussi une illustration d'un modèle créé de toutes pièces. Jusqu'à Champollion, les hiéroglyphes étaient simplement envisagés sous l'angle d'une cryptographie n'ayant pas vocation à être interprétée mais seulement considérée comme telle et révérée comme signe d'une intelligence supérieure.

\section{Le Journal für Freymaurer}

La floraison égyptomaniaque d'un grand nombre de temples maçonniques à l'époque de Mozart suscita parfois des dérives qui n'étaient pas du goût de Joseph II qui les qualifiait de "tours de passe-passe " (Gaukelei $\left.{ }^{18}\right)$. L'empereur, dans son entreprise de réforme de la franc-maçonnerie, s'était acquis la confiance d'Ignaz von Born (1742-1791), intellectuel influent, minéralogiste particulièrement bien vu par l'élite viennoise. Il a collaboré à cette restructuration à la fois réductrice mais aussi relativement saine puisque visant à extirper les " tours de passe-passe " qui, prenant de l'ampleur, pouvait profondément nuire à la Maçonnerie. I. von Born souhaitait instituer une Académie des sciences en réunissant les fleurons de l'intelligentsia maçonnique. Il fonda une revue qui devait se révéler d'une importance capitale pour comprendre l'identité et les orientations de la franc-maçonnerie: le Journal für Freymaurer. Sa diffusion était volontairement restreinte aux Frères. Le premier numéro parut en 1784 et comportait un article intitulé : Ueber die Mysterien der Egyptier ${ }^{19}$, rédigé par von Born. Otto Jahn ${ }^{20}$, éminent biographe de Mozart, ainsi qu'Edgar Istel, dans son importante monographie sur la franc-maçonnerie sous-jacente à la Zauberflöte ${ }^{21}$, insistent sur l'influence notable exercée sur l'entourage du compositeur par cette publication. Ignaz von Born y exprime clairement la volonté de sortir les rites égyptiens de l'aura fantaisiste dénigrée par Joseph II, dans laquelle ils étaient plongés à l'époque, pour en extraire ce qui était censé convenir à la culture maçonnique ${ }^{22}$. Dans un Handbillet du 11 décembre 1785, Joseph II avait écrit: « Die sogenannten Freimaurergesellschaften, deren Geheimnisse mir ebenso unbewußt sind, als ich deren Gaukeleien zu erfahren wenig vorwitzig

18. Kuéss, 200 Jahre Freimaurerei in Österreich, p. 70.

19. Journal für Freymaurer: als Manuskript gedruckt für Brüder und Meister des Ordens, hrsg. von den Brüdern der Loge zur Wahren Eintracht im Orient von Wien, ersten Jahrgangs erstes Vierteljahr, Wien, Christian Friedrich Wappler, 5784 [i.e. 1784], p. 15.

20. Otto Jahn, W.A. Mozart, Leipzig, Breitkopf und Härtel, 1859, vol. 4, p. 605.

21. Edgar Istel, Die Freimaurerei in Mozarts Zauberflöte, Berlin, A. Unger, 1928, p. 13.

22. Journal für Freymaurer, p. 25. 
jemals war... ${ }^{23}$ ". Von Born prend alors la défense des emprunts à la civilisation égyptienne, à sa religion en particulier:

Bald ward ich gewahr, wie ungerecht das Urtheil neuerer Geschichtschreiber (sic) sey, welche die ältesten Aegyptier nach den heutigen beurtheilten, und sie als ein rohes ungesittetes Volk, ihre Religion als ein Gewebe von Unsinn, ihre Priester als Possenspieler und Betrüger, ihre Kenntnisse und Geheimnisse als Thorheiten und Gaukeley ansahen. ${ }^{24}$

Il développe ce point de vue dans le chapitre suivant: Vergleichung der Zeremonien und Gebräuche der ägyptischen Mysterien mit denen der Maureren ${ }^{25}$.

Le hiéroglyphe reste à l'état de signe, de trace conduisant à la Lumière. La plupart des rites maçonniques en vigueur proposent une initiation purificatrice par les quatre éléments, visible dans la Flûte enchantée. Si maintenant la purification initiatique est partie prenante d'une réplique des Mystères antiques, cela signifie qu'une brèche est ouverte, par laquelle peut pénétrer la Gaukelei. C'est ce qui advint des loges constituées par les Maçons que von Born désirait évincer, non seulement par conviction personnelle mais aussi pour satisfaire les recommandations de Joseph II. Alors, pourquoi cette évocation réitérée des Mystères? Parce que le secret, et lui seul, permet de protéger le savoir, la connaissance de l'Un au tréfonds des pyramides. Cette connaissance, malgré son aspect plutôt ésotérique et peu conforme à la philosophie des Lumières, se veut résolument "scientifique". C'est le concept de wissenschaftliche Maurerey introduit par Franz Anton Kreil (1757-1838). Catholique, lecteur puis professeur de philosophie au Theresianum de Vienne en 1785, il fut Hofrat à Ofen (Buda), prit ouvertement la défense de la Révolution française et fut démis de ses fonctions en 1793. Dans les procès-verbaux des tenues de la Loge " Zur wahren Eintracht ", Kreil a fait état de son étude sur les Mystères égyptiens lors des deux tenues des 16 et 22 avril $1785^{26}$, auxquelles Mozart avait assisté et lors desquelles son père fut élevé aux grades de Compagnon et de Maître. Durant ces tenues, reprenant une réflexion sur le néoplatonisme ${ }^{27}$, il tint un discours portant le titre Über die Wissenschaftliche Maurerey, qualifiant celle-ci de "scientifisch". Dans le même essai, il fait allusion à l'" illumination de l'intérieur " (Erleuchtung von innen) protégée par le secret.

\section{La sous-jacence discrète du judéo-christianisme}

Quel est ensuite l'héritage israélite de cet épisode égyptien et comment le christianisme régnant a-t-il succédé à l'épisode mosaïque? Un autre penseur éminent de la Maçonnerie autrichienne, contemporain de Mozart, Carl Leonhard

23. Kuéss, 200 Jahre Freimaurerei in Österreich, p. 67.

24. Journal für Freymaurer, p. 25.

25. Ibid., p. 85.

26. Harald Strebel, Der Freimaurer Wolfgang Amadé Mozart, Stäfa, Rothenhäusler, 1991, p. 208-209.

27. Journal für Freymaurer..., zweyten Jahrgangs zweytes Vierteljahr, Wien, C.F. Wappler, 5785 [i.e. 1785], p. 46-49. 
Reinhold (1757-1823), Jésuite apostat, franc-maçon appartenant à la mouvance des Illuminati, gendre de Wieland, a étudié la relation entre l'apparition de la religio juive et l'évanescence des cultes de l'ancienne Égypte. Dans son essai intitulé Hebräischen Mysterien (1788), réédité en 2002 par J. Assmann, qui exerça une influence notable sur la Maçonnerie viennoise, il critique tour à tour les concepts d'élection du peuple juif et de religion révélée strictement à celui-ci, et surtout la déchéance des mystères égyptiens. Sans vouloir forcer le trait, tout se passe comme si le secret disparaissait devant la révélation; la Connaissance sombre à certains égards dans une sorte de trivialité. L'effort de son acquisition par le postulant puis l'initié dans le secret des Mystères, est gommé ; il prend son lecteur à témoin et tente de le persuader en ces termes :

... dass diese ganze Nation aus lauter Eingeweihten bestanden habe, dass die geheime Religion der Weisen bey ihr gemeine Volksreligion gewesen sey, und dass die Wahrheiten, die man fast in der ganzen übrigen Welt nur den besten und edelsten Menschen anzuvertrauen gewohnt war, der gemeinste Besitz des dümmsten und bösartigsten Pöbels wurde, der uns aus der ältern und neuern Geschichte bekannt ist. ${ }^{28}$

Il est bon de rappeler également que l'égyptianité de Moïse fut minorée par la tradition. Il convient de noter enfin que, dans la totalité du livret de la Flûte enchantée, les vocables Israel et Moses, intégraux ou leurs racines, sont absents. Le Temple de Salomon (dont le nom n'est également pas cité, pas plus que celui de l'architecte Hiram) reste en revanche crucial, lieu sacré de l'Initiation. Cependant, rien ne permet d'affirmer que l'édifice monumental situé à l'arrière-plan du frontispice d'Alberti (et décrit en tête de la scène 2 de l'acte II) soit le Temple du souverain, fils de David, ni que la figure humaine qui gît à terre soit la dépouille de son architecte, assassiné par les trois mauvais compagnons. Nous sommes dans l'illustration, dans la copie interprétative d'un contexte originel antique où l'Égypte apparaît au moins dans deux éléments: l'obélisque et l'acacia. Le reste est composite, certes significatif pour qui sait décrypter les symboles, mais singulièrement hétérogène. Si nous continuons d'examiner les occurrences du judéo-christianisme dans les sources susceptibles d'avoir influencé l'entourage de Mozart, il convient d'évoquer un article remarquable à cet égard du $\mathrm{n}^{\circ} 2$ du Journal der Freymaurer (1784) : "Über Analogie zwischen dem Christenthume der ersten Zeiten und der Freymaurerey, von Br. M $\therefore$ ch $\therefore$.r. ${ }^{29}$." Il fait l'éloge du christianisme primitif, dont le caractère souvent secret pouvait sous certains aspects l'assimiler à la Maçonnerie. Dans le même esprit que Reinhold, il porte, au terme de sa démonstration, un jugement très nuancé sur le degré de Connaissance associé à Loi mosaïque, concluant: "...daß im Christenthume eine Menge neue Glaubeslehren vorkommen, von denen das mosaische Bekenntnis nicht wußte ».

28. Bruder Decius [i.e. Leohard Reinhold], Die Hebräischen Mysterien oder die älteste religiöse Freymaurerey, Leipzig, G.J. Göschen, 1788, p. 39.

29. Il s'agit d'un certain "Bruder Michaeler " auquel font allusion plusieurs monographies de l'époque, dont: Ignaz Aurelius Fessler, Eleusinien des neunzehnten Jahrhunderts, Berlin, Frölich, 1803, p. 82. 
Il rappelle néanmoins la prééminence de la religion égyptienne, au point que : " ...der Vorrath ägyptischer, in Griechenland und Asien verarbeiteter Kenntnisse für die Bedürfnisse der christlichen Gnosis hinreichen konnte ${ }^{30}$ ».

\section{Le personnage de Papageno}

Il nous semble opportun maintenant de revenir sur cette figure emblématique de la comédie autrichienne évoquée en introduction, Hans Wurst, dont Papageno serait l'avatar. Celui-ci en effet fera, de manière assez inattendue, le lien entre un aspect bien particulier des mythèmes égyptiens relatif au sort fait à la féminité et d'autre part la marginalisation de celle-ci dans ce qu'il est convenu d'appeler la Maçonnerie d'adoption.

Hans Wurst et Papageno côtoient des personnages socialement élevés. Les princes de ce monde, auprès desquels ils font pâle figure, sont cependant de différente nature. Le comique incarné par Stranitzky ridiculise des personnalités inscrites dans l'Histoire, que seuls distinguent leur renommée ou leur pouvoir - comme César, Cicéron ou Achille - tandis qu'il s'attire la faveur d'un public hilare. Sous les traits de Schikaneder, Papageno séduira ou fera également sourire le parterre mais restera dans l'ombre d'un homme symbolique et anhistorique, Tamino, illuminé par sa spiritualité et non par les lumières factices du monde profane. Les deux compères amusent, le second s'inscrit dans la lignée du premier, mais dans une perspective qui nuance notablement la perception qu'ils suscitent.

L'Homme-Oiseau fait irruption après la mort du Serpent, figure récurrente qui, de manière ambivalente, incarnera tour à tour le Mal et son remède, sur le caducée d'Hermès par exemple.

La figure du Serpent, dans la première scène du premier acte de la Flûte enchantée, peut être suivie au fil des écrits antiques et contemporains du XVIII ${ }^{\text {e }}$ siècle: chez Hérodote, Diodore de Sicile et Plutarque, mais aussi dans l'histoire du roi Sethos de l'abbé Terrasson ${ }^{31}$. Cette fiction, évoquée précédemment, élaborée en 1731 par un ecclésiastique français érudit, eut un succès retentissant parmi les élites européennes de l'époque et fut traduit en plusieurs langues. Il est une bonne illustration de ce "remodelage " de la religion égyptienne et se présente comme une compilation ou un florilège d'extraits desdits auteurs antiques. Il influença significativement les contemporains de Mozart.

Examinons plus avant l'épisode du Serpent. Les Trois Dames le découpent en trois morceaux et sauvent ainsi Tamino. Dans le Traité d'Isis et d'Osiris de Plutarque, nous voyons Horus parti au secours de Thouéris, concubine de Typhon ${ }^{32}$. Ce dieu maléfique avait lancé à sa poursuite un serpent dans

30. Journal für Freymaurer..., zweyten Jahrgangs zweytes Vierteljahr, p. 59-60.

31. Jean Terrasson, Geschichte des egyptischen Königs Sethos, aus dem Französischen übersetzt von Matthias Claudius, Breslau, Löwe, vol. 1, 1777; vol. 2, 1778.

32. Typhon est une divinité diabolique et duelle, ennemi d'Osiris, de l'Un, assimilé à Seth, hippopotame mâle. Sa concubine, Thouéris, est la déesse, bénéfique, protectrice de l'accouchement. 
l'intention de la tuer, car elle avait pris le parti d'Horus. Celui-ci, fils posthume d'Osiris, occit alors le reptile avec l'aide de ses compagnons. En souvenir de cet acte glorieux, une corde est tranchée en plusieurs morceaux lors des cérémonies dans le Temple ${ }^{33}$.

Tamino, confondu, s'éveille :

Tamino (erwacht, sieht furchtsam umher) Wo bin ich? Ist‘s Fantasie, daß ich noch lebe? oder hat eine höhere Macht mich gerettet? (steht auf, sieht umher.) Wie? - Die bösartige Schlange liegt todt zu meinen Füßen? - (man hört von fern ein Waldflötchen, worunter das Orchester piano accompagnirt. Tamino spricht unter dem Ritornel.) Was hör ich? Wo bin ich? Welch` unbekannter Ort! -Ha, eine männliche Figur nähert sich dem Thal. (versteckt sich hinter einem Baume). ${ }^{34}$

La peur, la magie, l'obscurité. C'est au tréfonds de son identité que l'homme " hésitant", "postulant" sans le savoir encore, est saisi. Les Dames sont dépêchées par la Reine de la Nuit. Son valet va surgir: l'Oiseleur. Dans l'univers féminin qui régit le début du Singspiel gît un rapport à l'oiseau qui se réfère à ce personnage second sinon secondaire, incarné par Papageno. Il renvoie luimême à une "Maçonnerie secondaire " celle des femmes: la Maçonnerie dite d'adoption.

Papageno se définit alors comme un "attrape-oiseaux " (Vogelfänger): "Ich fange für die stern flammende Königinn und ihre Jungfrauen verschiedene Vögel $^{35}$."

Dualité encore: deux "religions ", deux genres (au sens physiologique), deux niveaux de compréhension. Le secret protège non seulement de la plèbe superstitieuse, mais aussi de la gent féminine comme l'indique explicitement un autre passage du Journal für Freymaurer dans sa première livraison de 1794, au chapitre 3 rédigé par von Born, dans un contexte spécifiquement égyptien : "Unsre weisen Gesetzgeber entfernten ebenfalls das schöne Geschlecht von unsern Geheimnissen ${ }^{36}$. "Ce qui vaut pour les secrets touche aussi les Mystères: "Das schöne Geschlecht war auf immer von den Mysterien ausgeschlossen ${ }^{37}$. "

Le nom d'Isis, divinité suprême, est évidemment prééminent, mais en aucun cas assimilable à une quelconque souveraine, encore moins à la Königin der Nacht. De souveraine, justement, il n'est point question. Certes, Hérodote ${ }^{38}$ mentionne la reine Nitocris mais, s'empresse d'ajouter I. von Born, elle incarne l'exception qui confirme la règle: "diese Ausnahme bestättiget selbst das allgemeine Grundgesetz ${ }^{39}$ ». Car enfın, les femmes au Temple sont reléguées à

33. Semler, Erleuterung der egyptischen Altertümer, p. 336-337.

34. Schikaneder, Die Zauberflöte, p. 3-4.

35. Ibid., p. 7.

36. Journal für Freymaurer... ersten Jahrgangs erstes Vierteljahr, p.93.

37. Ibid., p. 91.

38. Herodotos, Die Geschichten von Herodotos. Erster Teil: Klio, Euterpe, Thalia, Melpomene übersetzt von Friedrich Lange, neu hrsg. von Dr. Otto Güthling, Leipzig, Reklam, [um] 1885, p. 180.

39. Journal für Freymaurer... ersten Jahrgangs erstes Vierteljahr, p. 91. Le concept de " reine d'Égypte " est en soi diffıcile à cerner. Reste sans doute à déterminer la part de la misogynie 
des tâches d'" entretien ": " ...schränkte sich der ganze Dienst der Frauen bey den Mysterien auf die unbedeutende Beschäftigung ein, Käfer, Spitzmäuse und einige andere den Aegyptiern heilige Thiere zu füttern ${ }^{40}$. "

Cette relégation se retrouve dans une conception quelque peu marginalisée de la Maçonnerie "réservée " aux femmes; celles-ci sont "adoptées " par les hommes et non intégrées à proprement parler. Leur correspond un rituel spécifique, placé sous l'autorité des Frères. Certaines loges féminines sauront se distinguer et s'affirmer, notamment en France dès le milieu du XVIII ${ }^{\mathrm{e}}$ siècle $^{41}$. Ce qui nous incite à évoquer les loges d'adoption en lien avec l'oiseleur Papageno, c'est le rituel décrit dans un ouvrage français de l'époque traduit en allemand: La Vraie Maçonnerie d'adoption, de Louis Guillemain de Saint-Victor $(1779)^{42}$. L'examen du rituel d'initiation au quatrième grade contient des éléments significativement associés au thème de l'oiseleur : "Auf dem Altar steht eine Schüssel, in welcher ein umgekehrtes Gefäß sich befindet, unter welchem ein lebendiger Vogel versteckt ist ${ }^{43}$. " Au terme de ses épreuves, la postulante est invitée à "lever le vase ": " “...heben Sie dieses Gefä $\beta$ in die Höhe, und genießen das reine Vergnügen, welches jede tugendhafte Seele fühlt, indem sie Glückliche macht !”. Die Schwester gehorcht, und der Vogel fliegt davon ${ }^{44}$ ". Libéré, l'oiseau doit maintenant échapper à l'oiseleur envoyé par la Reine de la Nuit. Papageno est l'homme asservi par une femme qui veut usurper les Secrets. Sous des dehors niais et rassurants, il est le premier être humain que rencontre Tamino à la fin de l'épisode du serpent. C'est aussi un messager de la Reine de l'Ombre dont une Sœur parfaite se distinguera en libérant l'oiseau. Cet aspect singulier de l'analyse s'achève sur une note biblique avec le psaume 124, verset 7 : "Notre âme s'est échappée comme l'oiseau du filet des oiseleurs ; le filet s'est rompu et nous nous sommes échappés ${ }^{45}$. "Verset qui répond à cet autre:

C'est en l'Éternel que je cherche un refuge. Comment pouvez-vous me dire: " Fuis dans vos montagnes, comme un oiseau? ". Car voici, les méchants bandent l'arc, ils ajustent leur flèche sur la corde, pour tirer dans l'ombre sur ceux dont le cœur est droit ${ }^{46}$.

Certes dépourvu du trident meurtrier, Papageno serait plutôt un rétiaire qu'un archer. Il capture et retient les âmes pour la Reine de la Nuit que seules les

ambiante dans cette marginalisation.

40. Ibid., p. 93.

41. Françoise Moreillon, Le Rite d'adoption, Biennale culturelle maçonnique Bordeaux 13 mai 2012, en ligne: [http://imaq.fr/bcm2012-cr/reportage3-13.html] (lien audio en menu de marge droite, ligne supérieure ; consulté le 23 novembre 2015). Outre-Atlantique, à Boston vers 1778, Hannah Mather Crocker fonda la première loge féminine américaine, St Ann's Lodge.

42. Louis Guillemain de Saint-Victor, La Vraie maçonnerie d'adoption, précédée de quelques réflexions sur les loges irrégulières, à Londres, aux dépens de l'Auteur, 1779.

43. Louis Guillemain de Saint-Victor, "Die wahre Adoptions-Maurerey oder Handbuch der Freymaurerinnen mit vorläufigen Anmerkungen über unregelmäßige Logen ", in Vollständige Sammlung der ganzen Adon-Hiramitischen Maurerey, Leipzig, A.F. Böhmen, 1786, p. 231.

44. Ibid., p. 235.

45. La sainte Bible... éd. Louis Segond, Genève, Maison de la Bible, 1974.

46. Ibid., Ps. 11 v. 1 et 2. 
apparences assimilent de façon spécieuse à des forces maléfiques, lunaires et ténébreuses. Il est au service d'un processus qui le dépasse, il est un acteur nécessaire du Theatrum mundi. Dans la Weltanschauung de la Flûte enchantée, il est appelé à l'initiation mais son rôle assigné d'oiseleur, chasseur inconscient des conséquences obligées de ses actes, le confınera toujours à l'apparence de l'initiation, à son reflet inopérant, à sa pâle copie. Aimable rebelle, il doit exister, dans l'ombre de la Reine car il est écrit que les Ténèbres n'ont pas compris la Lumière. Mais c'est dans l'Ombre que chemine le postulant, et l'innocent Papageno chemine aux côtés du fier Tamino sans jamais le rejoindre, car tel est son destin (et pour partie, celui de certains spectateurs). Bouffon ou primesautier, il accompagne ce noble prince, de même que Mozart a son double qui choque " le monde ", cette société passablement élitiste au sein de laquelle se déploie la Maçonnerie. Mozart sera par ailleurs un Frère très honorable et respecté en loge, mais soucieux d'embrasser le tissu humain tout entier, sans circonscrire son art de vivre à celui de l'Atelier. C'était là sans doute pour lui une autre conception de la Fraternité, celle-là même qui l'unissait à von Born, Schikaneder, Giesecke, Alberti et tant d'autres et qui contribua à l'inspiration de la Zauberflöte.

\section{Utopie et miroirs}

De la paraphrase égyptienne à l'évocation des Psaumes, c'est une révolution spirituelle qui se manifeste, à travers les siècles, jusqu'au XVIII . L'égyptomanie dominante à l'époque dans une Maçonnerie que Joseph II souhaitait "purger " de ce qu'il avait nommé "Gaukelei ", a sans conteste teinté en contrepoint la forme de la Flûte enchantée. Le Journal für Freymaurer en témoigne. Néanmoins, nous voyons que son inspiration doit plus à l'adaptation d'un Urbild, classique à l'époque, celui de l'égyptianité, popularisé notamment par la fiction de l'abbé Terrasson, l'histoire de Sethos. On peut avec raison réfuter le caractère proprement égyptien des lieux du Singspiel. Il est manifestement utopique, même si son topos est associé sans conteste à l'univers pharaonique. Le propos, tant du livret que de la partition, reste au demeurant évocateur d'un rite et d'une tradition nilotiques. "Invocation " pourrions-nous dire, qui correspond à un jeu de miroirs. Il rend cet opéra tout à la fois contrefacteur et victime de sa propre contrefaçon. Incompris par les uns, il laisse affleurer, entre les lignes d'une partition riche en modulations tonales expressives, des concepts assez clairement interprétés par d'autres. 
